\title{
Long term periodontal problems - the chemotherapeutic aspect.
}

\section{R. M. Palmer}

\section{This is the fourth article in a series written by a selection of experts who will be speaking at this year's National Dental Conference: Best Practice 2001. In this issue, Professor R. M. Palmer explores what we should be telling our patients about long-term periodontal problems.}

$\mathrm{T}$ he majority of the population are not affected by chronic periodontitis to a degree that threatens the retention of their teeth. For these individuals their periodontal treatment requirements are minimal. The latest Adult Dental Health Survey (1998) reports that overall only 5\% of dentate adults have pocketing of $6 \mathrm{~mm}$ or more, although $9 \%$ of the 55-64 year olds and $15 \%$ of the over 65 year olds are affected. It is useful that the $6 \mathrm{~mm}$ and over category of pocketing was recorded in the ADHS as it is a commonly accepted level of pocketing at and above which treatment becomes more difficult.

The removal of plaque from the tooth surface remains the mainstay of periodontal treatment, regular supragingival plaque removal by the patient and subgingival cleaning by the dentist/hygienist. Many research papers have reported that the most dramatic reductions in pocketing occur with root planing of the deepest sites, but it is pertinent to recall that these sites are also more likely to end up with some residual pocketing. The clinician is then left with the decision whether to accept the residual pocket (and try to maintain it) or improve the result with other treatment modalities.

Antibiotics, both systemic and topical, are often used in an attempt to improve the clinical outcome. Clinical trials have shown that antibiotics may have an adjunctive effect, but the additional benefit is limited, on average less than $1 \mathrm{~mm}$ further reduction in probing depth. This result applies to both systemic and locally delivered antimicrobials. So why are they not more effective?

Richard M Palmer works at the Department of Periodontology and Preventative Dentistry at Guy's, King's and St Thomas' Hospital, London
Much emphasis is placed on the fact that the bacteria within the pocket exist as a plaque biofilm that adheres to the root surface. Antimicrobials penetrate the biofilm poorly and bacteria within it may be more resistant to antibiotics than the same species of bacteria growing in suspension or on laboratory media. The recommendation is therefore to disrupt the plaque biofilm by mechanical instrumentation before applying the antimicrobial. The antimicrobial may then be more effective against the residual bacteria, although reformation of a biofilm may occur quite rapidly. It is generally thought that it may take up to three months for the subgingival plaque biofilm to re-establish and this is one of the reasons for the commonly adopted maintenance interval of three months. However in some cases bacterial recolonisation may occur very much more rapidly.

Where does the subgingival biofilm reestablish from? This may occur from residual bacteria on the root surface within the pocket following instrumentation (missed by the clinician) or from a supragingival source. The latter is most likely to be due to inadequate plaque control by the patient but current theories also include re-infection by periodontal pathogens from other pockets, and other niches such as the dorsal surface of the tongue and tonsils. This has led to the concept of full mouth disinfection regimes to suppress the source of these reinfecting organisms.

Regimes include completing all subgingival instrumentation within a short time frame (e.g. 48 hours), subgingival irrigation with chlorhexidine, tongue brushing and extensive use of chlorhexidine mouthrinse. Antimicrobials can therefore be used in conjunction with biofilm removal to improve the chances of total bacterial clearance or to delay/prevent recolonisation (biofilm reformation).

These approaches may have merit in the initial management of advanced periodontitis, but how long can such approaches be continued in a maintenance programme that is for the life of the patient/dentition? Although antimicrobial mouthrinses such as chlorhexidine can be used for extensive periods of time, they do not penetrate subgingivally.

Repeated use of antibiotics is not to be recommended as it leads to emergence of resistant strains. Therefore they cannot be recommended for management of residual chronic periodontal disease. In conclusion, the main points relating to use of antimicrobials are:

- There is little or no justification for the use of systemic antibiotics in the treatment of the common forms of adult periodontitis.

- Application of topical or systemic antimicrobials without biofilm disruption is likely to be ineffective.

- Sites which are subjected to thorough subgingival debridement may show little additional benefit with adjunctive antimicrobials

- Long term repeated application of antimicrobials has not been adequately tested in clinical trials.

- The cost effectiveness and cost benefit of topical antimicrobial treatment is difficult to quantify

- Full mouth disinfection regimes will need further evaluation before general recommendation

The Best Practice 200 I

Conference will take place between 3-5 May 200I at the Harrogate International Centre. For a copy of the registration form please contact the Events Office on 02075634590 . 